\title{
Comparison of diagnostic performances of three different software packages in detecting coronary artery disease
}

\author{
Raffaele Giubbini • Francesco Bertagna
}

Published online: 27 August 2010

(C) Springer-Verlag 2010

Quantitation was and is the main diagnostic goal of nuclear medicine. The ability to measure a process or to quantify a disease reflects the capability of a diagnostic tool to understand the intimal mechanism of physiological processes. Observing or measuring are completely different attitudes and they make the difference between modern, evidence-based medicine and the medicine of the nineteenth century based on subjectivity of physicians rather than objectivity of data.

The measure of myocardial blood flow and the definition of coronary flow reserve [1] have defined a cultural background able to open new research perspectives to investigators interested in understanding the intrinsic mechanism of myocardial ischaemia and coronary artery disease.

Measuring itself, however, is necessary but not sufficient to provide effective clinical information if investigators and physicians are not aware about the robustness of the quantitative data provided by different diagnostic tools.

In nuclear cardiology, traditional quantitative measures can be categorized in hierarchical order according to the complexity of the measure: (1) relative (count/voxel), (2) absolute $\left(\mathrm{MBq} / \mathrm{cm}^{3}\right)$ and $(3)$ physiological $\left(\mathrm{cm}^{3} / \mathrm{s}\right.$ per $\left.\mathrm{g}\right)$. The pathophysiology of coronary blood flow and ischaemia

This Editorial Commentary refers to the article http://dx.doi.org/ 10.1007/s00259-010-1522-1

\section{R. Giubbini}

Nuclear Medicine, University of Brescia,

Brescia, Italy

R. Giubbini $(\varangle) \cdot$ F. Bertagna

Cattedra ed U.O. Medicina Nucleare,

Università e Spedali Civili - Brescia,

P.za Spedali Civili, 1,

25124 Brescia, Italy

e-mail: giubbini@med.unibs.it

e-mail: raffaele.giubbini@spedalicivili.brescia.it has been widely evaluated by absolute measures of blood flow and coronary flow reserve by positron emission tomography (PET) imaging with a variety of tracers including ${ }^{15} \mathrm{O},{ }^{13} \mathrm{NH}_{3}$ and ${ }^{82} \mathrm{Rb}$ and by standard nuclear medicine with ${ }^{99 \mathrm{~m}} \mathrm{Tc}$-microspheres. Other non-nuclear methods including fractional flow reserve and coronary flow velocity reserve have been widely validated to measure the physiological severity of a stenosis which may or may not determine myocardial ischaemia in subtended vascular territories. All these methods, however, require sophisticated technology, including PET, cyclotrons for production of shortlived isotopes or expensive rubidium generators, complicated analysis programs which not always guarantee high reproducibility or, when non-nuclear methods are applied, invasive tests. This is the reason why the use of absolute, physiologically relevant approaches are limited to a few academic centres and are mainly utilized for research purposes. Therefore simpler, cheaper and more easily applicable methods have been developed for evaluating myocardial ischaemia. Although unable to measure the coronary blood flow, the relative methods allow the calculation of extent and severity of perfusion defects in comparison to a normal database. The degrees of extent and severity of perfusion defects are integrated in an adimensional score that is an index of myocardial scar and/or ischaemia. These scores may vary according to physiological determinants (amount of myocardial ischaemia) and methodological criteria including type of tracer administered, kind of stressors, algorithm for single photon emission computed tomography (SPECT) reconstruction, software for quantification, left ventricular segmentation model and reliability of the normal database. In this issue of the European Journal of Nuclear Medicine and Molecular Imaging Guner et al. evaluate the performances of three of the most popular software packages adopted worldwide for "quantitative analysis" of myocardial perfusion imaging 
(MPI): the CEqual, the Emory Cardiac Toolbox and the 4DMSPECT [2]. In this retrospective study the authors investigate the diagnostic accuracy in a large cohort of subjects in comparison to coronary angiography to detect coronary disease using both the database of normal subjects provided by the commercial software and an institutional database collected at the Department of Nuclear Medicine, Gazi University School of Medicine, Besevler, Ankara, Turkey.

One of the points of interest in this study is the use of ${ }^{201} \mathrm{Tl}$. As underlined by the authors this tracer is cyclotron produced and easily available also in the present era of technetium shortage, but ${ }^{201} \mathrm{Tl}$ has, also, interesting physiological characteristics due to a higher extraction fraction than technetium-labelled perfusion tracers, although its less favourable physical properties and the high dosimetry for the patients have limited its clinical application in the last decades. The new gamma cameras with cadmium zinc telluride (CZT) detectors [3] could encourage renewed interest in the use of this tracer due to their very high count efficiency and, consequently, the possibility of limiting the administered activity. Furthermore, the use of stationary detectors could allow the registration of the input function and the calculation of absolute myocardial blood flow, having an accurate attenuation correction by computed tomography (CT).

Considering the semiquantitative analysis by perfusion score the authors have found significant variations in both summed stress score (SSS) and summed difference score (SDS) between the three different software packages, with the obvious consequence that for therapeutic implications and follow-ups their interchangeable use is prohibited. A second point of interest was the use of receiver-operating characteristic (ROC) analysis to determine different thresholds in the SSS, able to identify patients with coronary artery disease with the same level of accuracy. At the traditionally used SSS threshold of $\geq 4$, 4D-MSPECT revealed a sensitivity of $73.7 \%$ and specificity of $63.3 \%$. Similar values (sensitivity of $76.3 \%$ and specificity of 63.9\%) were obtained by Quantitative Perfusion SPECT (QPS) with SSS $\geq 9$ and by Emory Cardiac Toolbox with SSS $\geq 8$ (sensitivity of $74.6 \%$ and specificity of $66.9 \%$ ). Although in the "limitation of the study" paragraph Guner et al. correctly underline that the poor specificity of an SSS threshold of $\geq 4$ to predict coronary artery disease (CAD) could be related to the patient selection and to the wellknown post-test referral bias due to a preferential selection of patients with a positive test response for coronary angiography [4], they have raised the question of the reliability of thresholds in the MPI scoring system and classification, especially when we move from population to single patient analysis. From the nuclear trial [6] of the Clinical Outcomes Utilizing Revascularization and Aggressive Drug Evaluation (COURAGE) study [7] we have learnt that in stable CAD patients, knowledge of the ischaemic burden is helpful in therapy selection and risk assessment: patients with moderate-severe ischaemia in whom there is no ischaemia reduction after treatment have a worse prognosis. Percutaneous coronary intervention (PCI) added to optimal medical therapy resulted in greater ischaemia reduction and the benefit was greater in patients with more severe ischaemia. Therefore a $>5 \%$ ischaemia reduction or elimination of residual ischaemia should be a therapeutic target in CAD patients. A correct quantification of perfusion defect is therefore mandatory for patient management.

Finally a limitation not mentioned by the authors is that this study was conducted without attenuation correction. The majority of centres involved in nuclear cardiology imaging do not routinely perform attenuation correction, but, as pointed out by Garcia [5], attenuation correction is an essential tool for extracting quantitative parameters from all types of cardiac radionuclide distributions and will play an important role in establishing cardiac SPECT for flow imaging.

In any case Guner and colleagues have opened a door; they or other investigators have to complete the job to define precise spheres of application of commercial software for semiquantitative MPI analysis in order to increase their reliability in interpreting studies in everyday practice. Detection and description of myocardial ischaemia are not enough and a correct quantification of the ischaemic burden should be routinely applied in all laboratories performing nuclear cardiology studies.

\section{References}

1. Gould KL, Lipscomb K, Hamilton GW. Physiologic basis for assessing critical coronary stenosis. Instantaneous flow response and regional distribution during coronary hyperemia as measures of coronary flow reserve. Am J Cardiol 1974;33:87-94.

2. Guner LA, Karabacak NI, Cakir T, Akdemir OU, Kocaman SA, Cengel A, et al. Comparison of diagnostic performances of three different software packages in detecting coronary artery disease. Eur J Nucl Med Mol Imaging 2010.

3. Buechel RR, Herzog BA, Husmann L, Burger IA, Pazhenkottil AP, Treyer V, et al. Ultrafast nuclear myocardial perfusion imaging on a new gamma camera with semiconductor detector technique: first clinical validation. Eur J Nucl Med Mol Imaging 2010;37(4):773-8.

4. Rozanski A, Diamond GA, Berman D, Forrester JS, Morris D, Swan HJ. The declining specificity of exercise radionuclide ventriculography. N Engl J Med 1983;309(9):518-22.

5. Garcia EV. SPECT attenuation correction: an essential tool to realize nuclear cardiology's manifest destiny. J Nucl Cardiol 2007;14:16-24.

6. Shaw LJ, Berman DS, Maron DJ, Mancini GB, Hayes SW, Hartigan PM, et al. Optimal medical therapy with or without percutaneous coronary intervention to reduce ischemic burden: results from the Clinical Outcomes Utilizing Revascularization and Aggressive Drug Evaluation (COURAGE) trial nuclear substudy. Circulation 2008;117(10):1283-91.

7. Boden WE, O'Rourke RA, Teo KK, Hartigan PM, Maron DJ, Kostuk WJ, et al. Optimal medical therapy with or without PCI for stable coronary disease. N Engl J Med 2007;356(15):1503-16. 